\title{
Effect of Charge Separation on the Stability of Large Wavelength Fluctuations during Spinodal Decomposition
}

\author{
Catherine M. Bishop* R. Edwin García W. Craig Carter \\ Department of Materials Science and Engineering, Massachusetts Institute of \\ Technology, 77 Massachusetts Ave., Cambridge, MA 02139
}

\begin{abstract}
A stability analysis of phase separation of charged species by spinodal decomposition is presented. The charge effects introduce a short wave number cutoff for linear perturbations about the homogeneous, neutral solution. Phase field calculations using a semi-implicit spectral method support this conclusion. This suggests that coarsening is limited in ionic solid systems that are unstable with respect to chargedphase separation.
\end{abstract}

Key words: spinodal decomposition, stability, kinetics

\section{Introduction}

The classical approach to spinodal decomposition formulates the chemical instability of an alloy [1]. The theory predicts the conditions for and the characteristic length-scale of instability with respect to composition fluctuations. It is possible in ionic systems that a composition fluctuation would also induce a charge-density fluctuation which is not considered in the classical analysis. While deviations from electro-neutrality will always increase the electrostatic energy, it is conceivable that in some systems for which like-neighbor bonds are favored (such as with partially covalent bonding or van der Waals interactions) that small regions with nonzero charge density could be stable. The long-range effect of a periodic charge-density fluctuation would be similar to

* Corresponding author: Phone 617.258 .5551 Fax 617.258 .7874
Email address: cbishop@mit.edu (Catherine M. Bishop). 
a Madelung energy and therefore systems with large dielectric constant would be candidates for such charged instabilities.

There are unpublished experimental observations in partially-ionic systems that suggest that such charged composition fluctuations may exist[2]. The purpose of this paper is to analyze the conditions under which such fluctuations may appear and to examine the wavelength of the initial stability. Furthermore, because the energy will increase with the size of the charged domains, it should be expected that an upper bound for the size of the charged domain will exist. In this paper, the minimum stable size, the fasted growing wavelength, and the coarsening limit are predicted.

This paper presents theory, stability analysis, and phase-field simulations of the early stages of spinodal decomposition in an ionic solid with a comparison to simple chemical and elastic systems. The object of this paper is to present a new, thermodynamic approach to unstable ionic systems that incorporates Maxwell's equations naturally. We consider an ionic material with constituents $A^{+a}, B^{+b}$, and $C^{-c}$ that is chemically unstable with respect to fluctuations in cation concentration. The neutral composition is $A_{y_{0}} B_{1-y_{0}} C_{z_{0}}$ where $y_{0}$ is the fraction of $A^{+a}$ ions on the cation sites and $z_{0}$, the ratio of the number of anion to cation sites, is fixed. This work examines the stability of systems that are unstable with respect to fluctuations in $y$, the fraction of $A^{+a}$ ions on the cation sites, about $y_{0}$, the neutral fraction. Another paper addressing the application of a general thermodynamics-based approach to materials problems in electrically and magnetically active systems is in preparation [3]. The current work is consistent with this general approach.

After completion of the work, it was pointed out to the authors by L-Q. Chen and B. Stephenson that the same result had been derived in a different manner in a Ph.D. Thesis by K.M. Gupta [4]. ${ }^{1}$ The authors were unaware of the earlier work until that time. Gupta's thesis examines the effect of charge in spinodally decomposing ionic systems of three components.

Non-uniform composition profiles have been observed in glassy intergranular pockets in yttria-doped $\alpha$-alumina and in Molecular Dynamics simulations of calcium aluminosilicate intergranular films in $\alpha$-alumina $[5,6]$. In these systems in addition to the normal bulk interactions, there is a competition between surface adsorption of a particular ionic species and electrostatic interactions, Fig. 1. Therefore, the method that we illustrate below should be directly applicable to finite or constrained systems.

Other authors have considered the effects of charged boundaries and defects in ionic systems. A brief description of some of those treatments of the elec-

1 Gupta's work was not published elsewhere. The present paper extends Gupta's results with numerical simulations. 
a)

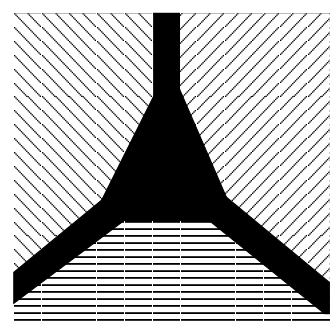

b)

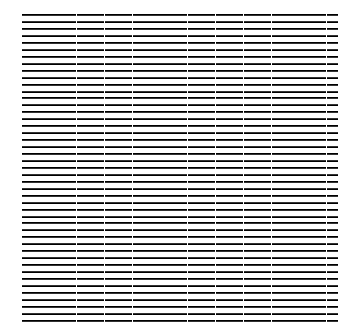

Fig. 1. Comparison of a) constrained, finite system (intergranular film and pockets) and b) infinite system (single crystal). The following analysis is applied to composition fluctuations in an infinite solid but the thermodynamics can be extended to treat constrained systems as well by including forces at the boundaries.

trostatic contributions to the energy is given for background and comparison with the method presented in this paper. The present treatment includes energetic contributions from externally applied as well as internally generated electric fields.

Yan et al. examine the relative strengths of elastic, electrostatic $\left(\frac{1}{2} \phi \rho\right)$ and dipolar $\left(-\frac{1}{2} \vec{P} \vec{E}\right)$ contributions to the free energy of a boundary region of a ceramic grain boundary [7]. The authors split up the electric contributions for the case that there are no externally applied fields.

Chen and Khachaturyan examine the effect of long-range Coulombic interactions during simultaneous microscopic ordering and phase separation [8]. The authors explicitly include a screened Coulomb potential with exponential form. Simulations suggest that the Coulombic term halts the coarsening in Fourier spectral space simulations at a finite wavelength. A typical scale for the decomposition is derived by minimizing the energy represented in spectral space with respect to wavenumber. In the present paper, neither the form of the electrostatic energy nor a cutoff is imposed.

Clarke et al. examine the effects of a double-layer on the stability of intergranular glassy films [9]. They use both DLVO theory and the counterion-only model to look at this contribution to the disjoining force while modeling the glassy film as a confined electrolyte. The authors' approach doesn't include an energetic description of the system, but instead considers only forces on a planar boundary.

This paper presents a stability analysis and confirming phase-field simulations for a ternary ionic material that is unstable to fluctuations in cation site fraction implying local deviations from neutrality at the temperature of interest. The general theory and stability analysis for systems of this type is presented in the Theory Section. The results of the stability analysis are discussed along with the simulations later in the Discussion section. 


\section{Theory}

The differential of the internal energy of a system with charged species is given by Eq. 1 where the natural variables, $S, V, n_{i}, q$, and $\vec{D}$, are entropy, volume, number of atoms of the $i$ th species, the total charge, and the electric displacement vector, respectively. The conjugate intensive variables, $T, P, \mu_{i}$, $\phi$, and $\vec{E}$, are temperature, pressure, chemical potential of the $i$ th species, electric potential, and applied electric field, respectively.

$$
d U\left(S, V, n_{i}, q, \vec{D}\right)=T d S-P d V+\mu_{i} d n_{i}+\phi d q+V_{0} \vec{E} \cdot d \vec{D}
$$

The internal energy is a first order homogeneous function of its natural variables. In the above, the electric work is split into two contributions: that from the charge in the system, $\phi d q$, and that from the total applied fields, $V_{0} \vec{E} \cdot d \vec{D}$, where $V_{0}$ is a stress-free reference volume. This splitting allows the model to account for electric potential resulting from the charge distribution within the system as well as any superposed externally imposed potential gradient.

For the analysis of materials at fixed $T, V, n_{i}, q$, and $\vec{E}$, the appropriate thermodynamic state function that is minimized at equilibrium is a partial Legendre transform of the internal energy $F=U-T S-V_{0} \vec{E} \cdot \vec{D}$, the Helmholtz free energy, given in differential form below [10].

$$
d F\left(T, V, n_{i}, q, \vec{E}\right)=-S d T-P d V+\mu_{i} d n_{i}+\phi d q-V_{0} \vec{D} \cdot d \vec{E}
$$

This function is homogeneous degree one in $V, n_{i}$, and $q$. The Helmholtz free energy results from integrating this function from an initial state to an arbitrary state, Eq. 3. The conjugate variables are, in general, functions of all of the natural variables for $F$, e.g., $\mu_{i}=\mu_{i}\left(n_{i}, q, \vec{E}, T, V\right)$. But as $F$ is a state function each integration is performed in succession to incrementally generate the total free energy by holding all other natural variables constant. The stress-free reference state is taken to have the temperature and volume of interest, so these become parameters in this problem.

$F\left(n_{i}, q, \vec{E} ; T_{0}, V_{0}\right)=F_{0}\left(n_{i, 0}, q_{0}, \vec{E}_{0}, T_{0}, V_{0}\right)+\int_{n_{i, 0}}^{n_{i}} \mu_{i} d n_{i}^{\prime}+V_{0} \int_{q_{0}}^{q} \phi d q^{\prime}-V_{0} \int_{\vec{E}_{0}}^{\vec{E}} \vec{D} \cdot d \vec{E}^{\prime}$

A linear constitutive relation between $\vec{D}$ and $\vec{E}, \vec{D}=\stackrel{\leftrightarrow}{\epsilon} \vec{E}$, where $\stackrel{\leftrightarrow}{\epsilon}$ is the dielectric permittivity tensor, gives Eq. 4 and dividing by the reference volume gives the Helmholtz free energy density, Eq. 5 .

$$
\begin{gathered}
F\left(n_{i}, q, \vec{E} ; T_{0}, V_{0}\right)=F_{0}+\mu_{i} n_{i}+\phi q-\frac{1}{2} V_{0} \vec{E} \cdot \stackrel{\leftrightarrow}{\epsilon} \vec{E} \\
\tilde{F}\left(c_{i}, \rho, \vec{E} ; T_{0}\right)=\tilde{F}_{0}+\mu_{i} c_{i}+\phi \rho-\frac{1}{2} \vec{E} \cdot \stackrel{\leftrightarrow}{\epsilon} \vec{E}
\end{gathered}
$$

Above, $\tilde{F}_{0}$ is the free energy density of the reference state, $c_{i}$ is the concentrations of the $i$ th species in moles $/ \mathrm{m}^{3}$, and $\rho$ is the charge density in $\mathrm{C} / \mathrm{m}^{3}$. 
Using the assumption of local equilibrium, the Helmholtz free energy density for a heterogeneous system is represented as a Taylor expansion of the homogeneous free energy density in gradient terms, Eq. $6[1,11,12]$. The thermodynamic variables are allowed to vary with position and the local values are representative of macroscopically small but microscopically large volumes such that these are appropriate to calculate the local contribution to the free energy. The gradient energy terms, where $\overleftrightarrow{\kappa}_{i}$ is the gradient energy coefficient tensor for the $i$ th species, intrinsically account for the interfacial free energy of internal diffuse interfaces. Assuming negligible magnetic effects, $\nabla \times \vec{E}=0$ and $\vec{E}=-\nabla \phi$. The total free energy of the system is the integral of the free energy density over the volume, $\Omega$.

$F\left[c_{i}, \rho, \phi ; T_{0}, V_{0}\right]=\int_{\Omega}\left[\left\{\tilde{F}_{0}+\mu_{i} c_{i}+\phi \rho-\frac{1}{2} \nabla \phi \cdot \stackrel{\leftrightarrow}{\epsilon} \nabla \phi\right\}+\left\{\frac{1}{2} \nabla c_{i} \cdot \overleftrightarrow{\kappa_{i}} \nabla c_{i}\right\}\right] d V$

The electrostatic free energy term has a similar form to the first order gradient energy terms with a negative gradient energy coefficient, $-\stackrel{\leftrightarrow}{\epsilon}$. However, its origin is the integration of the differential electrostatic enthalpy $\left(-V_{0} \vec{D} \cdot d \vec{E}\right)$, while the gradient terms derive from a Taylor expansion about a homogeneous system in gradients of the order parameters. A discussion of why this term doesn't lead to infinitely large gradients in $\phi$ is given below.

By considering an infinite three-component ionic solid composed of $A^{+a}, B^{+b}$, and $C^{-c}$ possessing at least cubic symmetry, the tensors reduce to scalars. Let the average composition of the solid be $A_{y_{0}} B_{1-y_{0}} C_{z_{0}}$ so that in one formula unit the neutral solution gives the following relation: $-a y_{0}+-b\left(1-y_{0}\right)+c z_{0}=0$. While requiring global charge neutrality, relaxing the restriction of local charge neutrality permits the possibility of the formation of charged domains. The local number density of electrons is $\sigma(\mathbf{x})$ given by $\sigma(\mathbf{x})=y(\mathbf{x})(b-a)+\left(c z_{0}-b\right)$. This is a rescaling that allows the local charge density to be written as a linear function of the local occupation of cation sites by $A^{+a}$ that is convenient for the following mathematics.

For example, consider bulk $\mathrm{MgTiO}_{3}$ or $\mathrm{Mg}_{y_{0}} \mathrm{Ti}_{1-y_{0}} \mathrm{O}_{1.5}$. The local excess electron density is zero when $y(\mathbf{x})=y_{0}=0.5$. When the local cation site fraction is $y(\mathbf{x})=0$, the composition is $\mathrm{TiO}_{1.5}^{+1}$ and the number density of electrons is $\sigma(\mathbf{x})=-1$. When the local cation site fraction is $y(\mathbf{x})=1$, the composition is $\mathrm{MgO}_{1.5}^{-1}$ and $\sigma(\mathbf{x})=1$.

The charge density is $\rho(\mathbf{x})=-\mathcal{F} \sigma(\vec{x})$ where $\mathcal{F}$ is Faraday's constant or the magnitude of the charge of a mole of electrons. The model system has fixed $z(\mathbf{x})=z_{0}$ and is unstable with respect to fluctuations in $y(\mathbf{x})$. The chemical contribution to the homogeneous free energy density at fixed volume, temperature, and anion concentration is a function of only the charge (or cation site occupation density, $y(\mathbf{x})$ ) and has the following form, Fig. 2. 


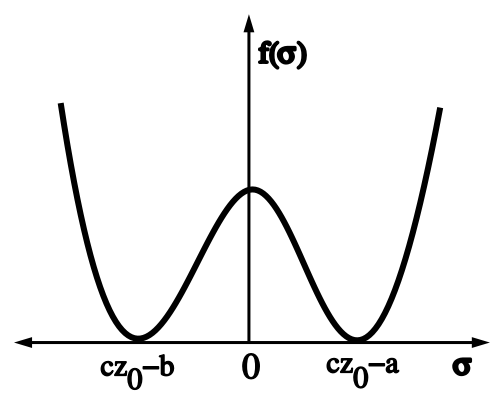

Fig. 2. Schematic of the chemical contribution to the homogeneous free energy, $f(\sigma)$, as a function of number density of electrons, $\sigma$, for a ternary system with fixed anion concentration and variable site fraction of cations, $y$. The maxima corresponds to the neutral composition and the minima to charged structural units. Because the charge is linearly related to the site fraction of cations, $y, f(y)$ has a similar but rescaled double well form.

The constraint of global mass conservation, $0=\int_{\Omega} \sigma(\mathbf{x}) d V$, is included with a constant Lagrange multiplier, $\lambda$, so that Eq. 7 takes the following form where $f(\sigma)=\tilde{F}_{0}+\mu_{A} c_{A}+\mu_{B} c_{B}+\mu_{C} c_{C}$.

$$
F[\sigma, \phi]=\int_{\Omega}\left[\left\{f(\sigma)-\mathcal{F} \sigma \phi-\frac{1}{2} \epsilon \nabla \phi \cdot \nabla \phi+\lambda \sigma\right\}+\frac{1}{2} \kappa|\nabla \sigma|^{2}\right] d V
$$

An equilibrium state of the system is described by the condition $\delta F=0$ where $\delta F$ represents a variation in the free energy [13]. In this case, there are two independent field parameters, $\sigma$ and $\phi$. A necessary condition for a weak extremum in the free energy for the fixed end-point problem is given by the Euler-Lagrange equations in Eq. 8 and Eq. 9.

$$
\begin{gathered}
\frac{\delta F}{\delta \sigma}=0=\frac{d f}{d \sigma}-\mathcal{F} \phi-\kappa \nabla^{2} \sigma+\lambda \\
\frac{\delta F}{\delta \phi}=0=-\mathcal{F} \sigma+\epsilon \nabla^{2} \phi
\end{gathered}
$$

The electric potential is assumed to be in steady state (instantaneous equilibrium with the composition field.) Therefore, Eq. 9 is always satisfied. This is Coulomb's Law that states that the charge density is the source of electric field.

The stability of an extremal solution can be determined by analyzing the stability of the appropriate kinetic equation for $\sigma$. As mass is conserved, $\sigma$ is a conserved order parameter and the appropriate kinetic equation is the Cahn-Hilliard equation, Eq. 10, where $M$ is a mobility [14].

The Lagrange multiplier is the electro-chemical potential of the reservoir and 
doesn't appear in the kinetic equation.

$$
\frac{\partial \sigma}{\partial t}=M\left[\nabla^{2} \frac{d f}{d \sigma}-\mathcal{F} \nabla^{2} \phi-\kappa \nabla^{4} \sigma\right]
$$

Substitution of Coulomb's Law, Eq. 9, yields

$$
\frac{\partial \sigma}{\partial t}=M\left[\nabla^{2} \frac{d f}{d \sigma}-\frac{\mathcal{F}^{2}}{\epsilon} \sigma-\kappa \nabla^{4} \sigma\right]
$$

Introducing non-dimensional parameters, $\xi, \tau$, and $\gamma$, with $\vec{x}=\vec{\xi}\left(\frac{\kappa}{f_{0}}\right)^{\frac{1}{2}}, t=$ $\tau\left(\frac{\kappa}{M f_{0}^{2}}\right)$ and $\gamma=\frac{\mathcal{F}^{2} \kappa}{f_{0}^{2} \epsilon}$. Here, $f_{0}$ is the magnitude of the curvature of $f(\sigma)$ at the consulate point, $f_{0}=\left|\frac{d^{2} f}{d \sigma^{2}}\right|_{\sigma=\sigma_{0}}$. The working form of the kinetic equation is Eq. 12.

$$
\frac{\partial \sigma}{\partial \tau}=\frac{1}{f_{0}} \nabla^{2} \frac{d f}{d \sigma}-\gamma \sigma-\nabla^{4} \sigma
$$

The kinetic equation for the initial stages of decomposition is obtained by expanding the free energy density, $f(\sigma)$, to second order about the consulate point and introducing a one-dimensional perturbation in composition about $\sigma_{0}=0$ along the $\xi_{1}$-direction with small time-dependent amplitude, $A(\tau, \beta)$, and dimensionless wave number $\beta$.

$$
\sigma\left(\tau, \xi_{1}\right)=\sigma_{0}+A(\tau, \beta) \sin \beta \xi_{1}
$$

Substitution of the Taylor expansion and Eq. 13 into Eq. 12 gives Eq. 14 for the time dependence of the amplitude of the Fourier mode with wave number $\beta$.

$$
\frac{d A}{d \tau}=A\left[\beta^{2}-\gamma-\beta^{4}\right]
$$

The form of $A(\tau, \beta)$ that satisfies Eq. 14 is given below in Eq. 15 where $R(\beta)$ is the growth factor.

$$
\begin{gathered}
A(\tau, \beta)=A(0, \beta) \exp [R(\beta) \tau] \\
R(\beta)=\beta^{2}-\gamma-\beta^{4}
\end{gathered}
$$

If the growth factor is positive (the perturbation is stable) then the amplitude of a fluctuation will grow in time. However, if the growth factor is negative (the perturbation is unstable) the amplitude will decay in time. In the electrochemical case there are maximum and minimum wave number cutoffs, $\beta_{\text {chem }}$ and $\beta_{\text {elec }}$, beyond which the system is stable with respect to perturbations. The critical values of the wave numbers, $\beta_{\text {elec }}$ and $\beta_{\text {chem }}$ satisfy $R(\beta)=0$, the static case. Figure 3 is a graphical representation of the results of the linear 
stability analysis for short times. The stability limits are given by the roots of Eq. 16 where $\beta_{\max }^{2}=\frac{1}{2}$ is the wave number corresponding the the maximum $R(\beta)$.

$$
\left(\frac{\beta}{\beta_{\max }}\right)^{2}=1 \pm \sqrt{1-4 \gamma}
$$

These stability limits may be compared to those from analyses by Cahn and Hilliard of the chemical case and Cahn of the elasto-chemical case $[1,15]$. In the second of these, Cahn assumes that the molar volume is a linear function of composition so that a composition fluctuation introduces a corresponding strain energy fluctuation. A stability analysis shows that the effect of the elastic terms is to alter the shape of $R(\beta)$ so as to reduce the $\beta_{\max }$ and the maximum value of $R(\beta)$. It is straightforward to include strain effects in the present scheme to consider the stability of an ionic material with a size mismatch between the ions to small perturbations in composition.

a)

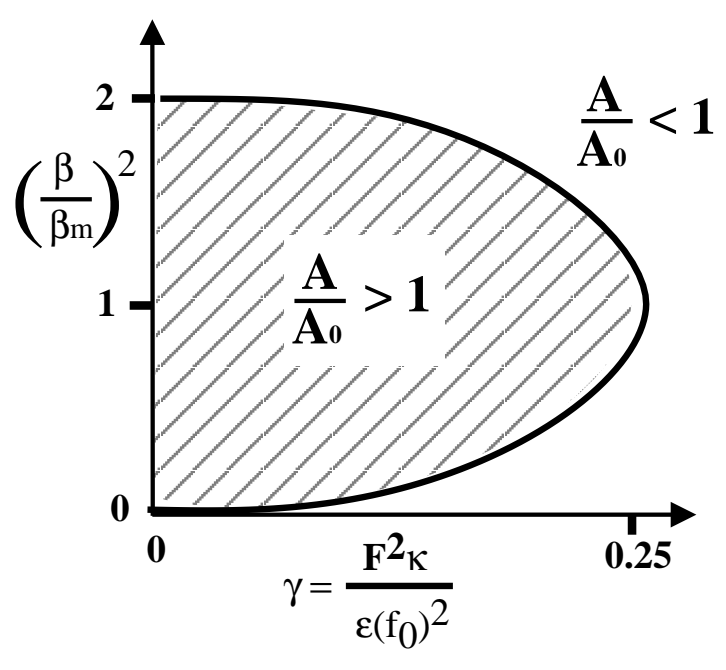

b)

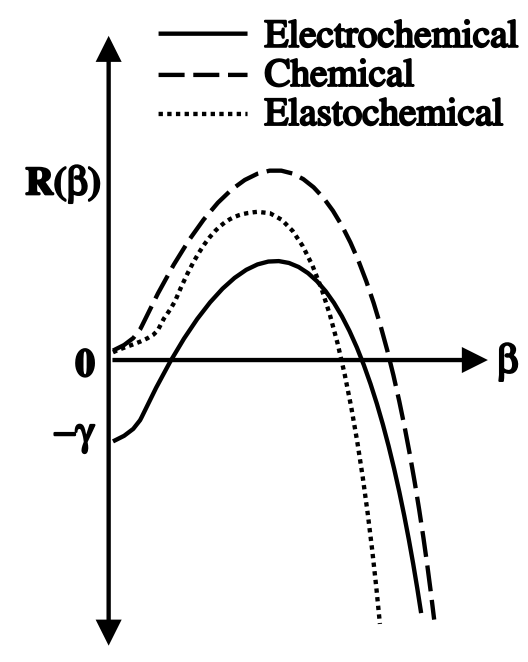

Fig. 3. Depiction of stability limits of an unstable ionic system. a) For a given value of $\gamma$ the amplitude of Fourier components with normalized wave numbers corresponding to the interior area grow in time. b) Wave Numbers with $R(\beta)>0$ grow in time. The present case (electro-chemical) is compared to two other previously analyzed cases (chemical and elasto-chemical).

\section{Simulations}

The kinetic equation, Eq. 12, was simulated on a periodic two-dimensional ionic system like $\mathrm{Mg}_{0.5} \mathrm{Ti}_{0.5} \mathrm{O}_{1.5}$ that is unstable to small fluctuations in cation site occupation in the absence of Coulombic effects. Therefore, the free energy is written as a quartic function given by $f(\sigma)=\frac{f_{0}}{4}\left(\sigma^{2}-1\right)^{2}$. The dimensionless kinetic equation for the composition field, Eq. 12, transformed to Fourier space is given in Eq. 18 where ${ }^{\wedge}$ indicates the Fourier transform, $\hat{\sigma}$ is the amplitude of each Fourier mode, $g(\sigma)=\frac{1}{f_{0}} \frac{d f}{d \sigma}=\sigma^{3}-\sigma$, and $k=|\vec{k}|=\left|k_{x} \hat{\imath}+k_{y} \hat{\jmath}\right|$ is the 
magnitude of the wave vector.

$$
\frac{d \hat{\sigma}}{d \tau}=-k^{2} \widehat{g(\sigma)}-\gamma \hat{\sigma}-k^{4} \hat{\sigma}
$$

A semi-implicit, Fourier-spectral scheme where the derivatives are accurate in space and first order accurate in time is employed [16].

$$
\left.\hat{\sigma}^{n+1}\left(1+\Delta \tau\left(\gamma+k^{4}\right)\right)=\hat{\sigma}^{n}-\delta \tau k^{2} \widehat{g(\sigma)}\right)^{n}
$$

The simulation parameters are $n_{x}=n_{y}=200, \Delta \tau=10^{-2}$, and the grid spacing (half the shortest wavelength) is $\Delta \xi=\frac{\lambda_{\min }}{2}=0.1$. The initial condition is assigned in real space according to $\sigma(\vec{x})=0.05(2 r-1)$ where $r$ is a random number from a uniform distribution, $0 \leq r \leq 1$, then transformed into spectral space. However, the magnitudes of the initial Fourier amplitudes are not part of a uniform random distribution but exhibit a maximum around $k=100$. This effect damps out after a very short time. Simulations with different values of $\gamma$ were performed, $0 \leq \gamma \leq 0.25$. The simulation results are presented as the amplitude of each Fourier component versus the magnitude of the wavevector for different times during simulations for the pure chemical case with $\gamma=0$ and for the electro-chemical case with small dielectric constant or large gradient energy, $\gamma=0.23$, in Fig. 4 .

a)

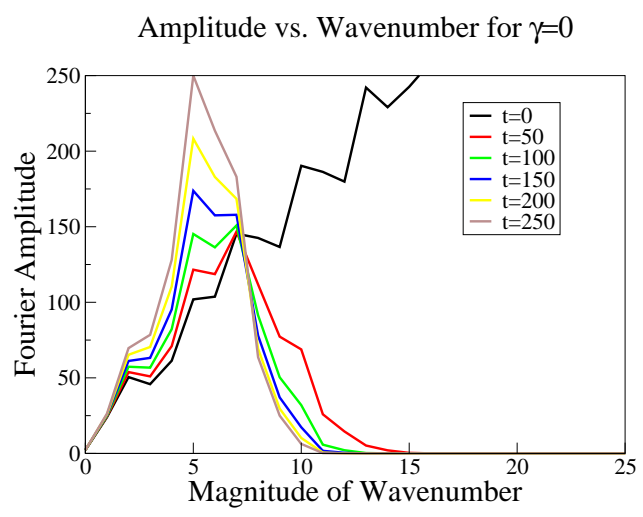

b)

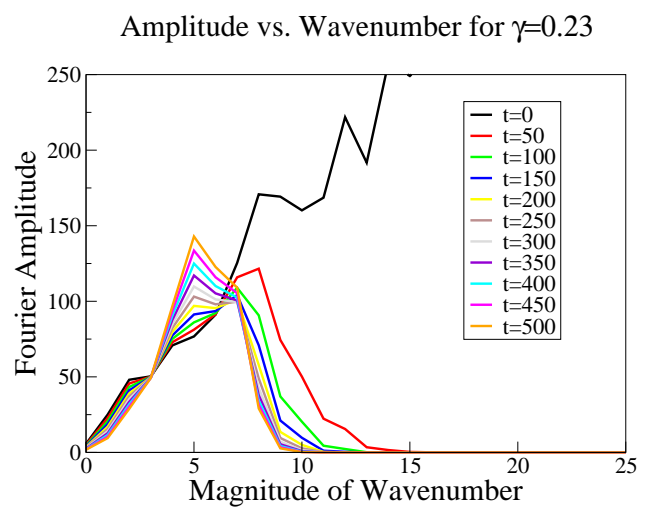

Fig. 4. Graphs of the amplitude of each Fourier component against the magnitude of each wave number for different number of iterations for experiments with $n_{x}=n_{y}=200, N=40$ and $\Delta \tau=10^{-2}$ with a) $\gamma=0$ b) $\gamma=0.23$. The maximum magnitude of the wave number in this work is $k_{\max }=100 \sqrt{2}$, but these plots focus on the low wave numbers as the large wave numbers damp out very fast. Both plots show approximately the same magnitude of the fastest growing wave number, but the electro-chemical system takes longer to reach this state.

\section{Discussion}

In this section, the stability of this electro-chemical system is compared to chemical and elasto-chemical systems. For the electro-chemical case, there 
is a range of growing wave numbers within the bounds determined by the undercooling, effective permittivity of the material, and the gradient energy coefficient. Figure 3 shows the normalized range of values of $\beta$ corresponding to growing amplitudes. Because $\gamma \propto 1 / \epsilon$, small values of $\gamma$ are representative of large values of $\epsilon$. This case approaches the chemical limit where $\left(\frac{\beta_{\text {chem }}}{\beta_{\text {max }}}\right)^{2}=2$ and $\left(\frac{\beta_{\text {chem }}}{\beta_{\text {max }}}\right)^{2}=0$, consistent with Fig. 3. If $f_{0}$ is small, then $\gamma$ approaches 0.25 and there is a small range of wave numbers that are unstable.

The maximum or fastest initial growing wave number in the electro-chemical case $\beta_{\max }=\frac{1}{2}$ is the same as the maximum growing wave number in the pure chemical case. The amplification factor for the electro-chemical case is shifted by a constant equal to $-\gamma$ with respect to the chemical case. For $\gamma \geq 0.25$, there are no wave numbers corresponding to growing amplitudes. This means that for short times the system is stable with respect to fluctuations of all wave numbers within this linear analysis. For $0 \leq \gamma<0.25$ (large dielectric constant) there is a short wave number cutoff in the electro-chemical case. This cutoff comes about from the electrostatic energy of charged regions separated by large distances. In the energy functional, Eq. 6, this is the third term contributing like a gradient energy penalty even though its origin is the homogeneous free energy term. A gradient energy term with a negative coefficient might be expected to induce infinitely large gradients in the corresponding independent field in order to minimize the free energy functional at equilibrium. However, that is not the case here because the values of the corresponding independent parameter, $\phi$, are coupled to the composition field through $\epsilon$.

The plots of the Fourier amplitudes, Fig. 4, as a function of wave number for the chemical case $(\gamma=0)$ and an electro-chemical case $(\gamma=0.23)$ support the above conclusions. The large wave number modes decay most rapidly for both cases. For the chemical case, all wave numbers below some critical value grow at first. In contrast, for the electro-chemical case, small wave number modes decay as well as large wave number modes so that the system doesn't coarsen beyond a cutoff wave number. This suggests that coarsening will be limited in the electro-chemical case; this is in contrast to the elasto-chemical case where domains of arbitrarily large size will develop. Also, the amplitude of the fastest growing mode is larger for the chemical case than for the electro-chemical case at any given time, compare Fig. 4 a) $\mathrm{t}=100$ to Fig. $4 \mathrm{~b}) \mathrm{t}=500$. This effect is also illustrated in Fig. 3 where $R\left(\beta_{\max }^{\text {chem }}\right)>R\left(\beta_{\text {max }}^{\text {electrochem }}\right)$. In addition, the initial curves for the data exhibit sharp peaks which smooth out in time as the distribution of stable wave numbers narrows. 


\section{Conclusions}

The Helmholtz free energy functional for a system with separate terms for the electrostatic work due to introducing charges into the system and for polarizing the medium can be used to model the instability in an ionic system. This functional is defined for a system at constant $T, V, n_{i}, q$, and $\vec{E}$, but the extension to other conditions is straightforward. A linear stability analysis for a model ternary system of ions decomposing into $A^{+a}$-rich and $B^{+b}$-rich domains due to small fluctuations in cation site occupation shows that, unlike the pure chemical case, there is a small wave number cut-off below which the system is stable with respect to fluctuations in cation site occupation. This conclusion was confirmed with numerical calculations. The results suggest that for given undercoolings, dielectric permittivities, and gradient energy coefficients decomposing systems will cease to coarsen beyond a cutoff scale given by $\beta_{\text {electrochem }}$.

This analysis suggests that charged domains may be observed in some ionic systems and quantifies the material properties that are required for their existence. It also presents microstructural scales that can be used to compare with experimental observations. The behavior of charged domains in an alternating electric field may significantly influence the behavior of ferroelectric materials. The behavior of model systems as a function of the frequency of an applied field remains an interesting problem.

\section{Acknowledgments}

The authors gratefully acknowledge the Singapore-MIT Alliance and NSF Grant DMR-0010062 for funding. We thank L.-Q. Chen and B. Stephenson for pointing out the similarity of our work with Gupta's thesis. We would also like to thank A.N. Soukhojak for sharing his unpublished results, R.M. Cannon, Y.-M. Chiang and J.W. Cahn for helpful discussions, and R.A. Kemper for proofreading this paper.

\section{References}

[1] J. W. Cahn and J. E. Hilliard, "Free energy of a non-uniform system. I. Interfacial free energy," J. Chem. Phys., vol. 28, pp. 258-267, 1958.

[2] A. Soukhojak, 2000. Private communication. 
[3] R. E. García, C. M. Bishop, and W. C. Carter, "Thermodynamically consistent variational principles with applications to electrically and magnetically active systems." in preparation, 2002.

[4] K. M. Gupta, Theory of continuous diffusional transformations and resulting charge-density waves in ionic systems. PhD thesis, Department of Materials Science and Engineering, Massachusetts Institute of Technology, 1976.

[5] M. Gulgun, 2000. Private communication.

[6] D. A. Litton and S. H. Garofalini, "Molecular dynamics simulations of calcium aluminosilicate intergranular films on (0001) $\mathrm{Al}_{2} \mathrm{O}_{3}$ facets," Journal American Ceramic Society, vol. 83, pp. 2273-2281, 2000.

[7] M. F. Yan, R. M. Cannon, and H. K. Bowen, "Space charge, elastic field, and dipole contributions to equilibrium solute segregation at interfaces," $\mathrm{J}$. Appl. Phys., vol. 54, pp. 764-778, 1983.

[8] L. Q. Chen and A. G. Khachaturyan, "Dynamics of simultaneous ordering and phase separation and effect of long-range coulomb interactions," Physical Review Letters, vol. 70, pp. 1477-1480, 1993.

[9] D. R. Clarke, T. M. Shaw, A. P. Philipse, and R. G. Horn, "Possible electrical double-layer contribution to the equilibrium thickness of intergranular glass films in polycrystalline ceramics," J. Am. Ceram. Soc., vol. 76, pp. 1201-1204, 1993.

[10] P. H. Badger, Equilibrium Thermodynamics. Boston: Allyn and Bacon, Inc., 1967.

[11] J. W. Cahn, "Free energy of a non-uniform system. II. Thermodynamic basis," J. Chem. Phys., vol. 30, pp. 1121-1124, 1959.

[12] E. W. Hart, "Thermodynamics of inhomogeneous systems," Phys. Rev., vol. 113, pp. 412-416, 1959.

[13] I. M. Gelfand and S. V. Fomin, Calculus of Variations. Mineola, New York: Dover Publications, Inc., 1963.

[14] W. C. Carter, J. E. Taylor, and J. W. Cahn, "Variational methods for microstructural-evolution theories," JOM, vol. 49, pp. 30-36, 1997.

[15] J. W. Cahn, "On spinodal decomposition," Acta Metallurgica, vol. 9, pp. 795$801,1961$.

[16] L.-Q. Chen and J. Shen, "Applications of semi-implicit fourier-spectral method to phase field equations," Computer Physics Communications, vol. 108, pp. 147$158,1998$. 